\title{
THE N2K CONSORTIUM. I. A HOT SATURN PLANET ORBITING HD $88133^{1}$
}

\author{
Debra A. Fischer, ${ }^{2}$ Greg Laughlin, ${ }^{3}$ Paul Butler, ${ }^{4}$ Geoff Marcy, ${ }^{5}$ John Johnson, ${ }^{5}$ Greg Henry, ${ }^{6}$ Jeff Valenti, ${ }^{7}$ \\ Steve Vogt, ${ }^{3}$ Mark Ammons, ${ }^{3}$ Sarah Robinson, ${ }^{3}$ Greg Spear, ${ }^{3} \mathrm{~J}_{\text {ay }}$ Strader, ${ }^{3}$ Peter Driscoll, ${ }^{2}$ \\ Abby Fuller, ${ }^{2}$ Teresa Johnson, ${ }^{2}$ Elizabeth Manrao, ${ }^{2}$ Chris McCarthy, ${ }^{2}$ Melesio Muñoz, ${ }^{2}$ \\ K. L. Tah, ${ }^{2} \mathrm{~J}_{\text {ASON Wright }}{ }^{5}$ Shigeru Ida, ${ }^{8}$ Bun'ei Sato, ${ }^{9}$ \\ ERi Toyota, ${ }^{9}$ And DanTe Minniti ${ }^{10}$ \\ Received 2004 September 7; accepted 2004 October 12
}

\begin{abstract}
The N2K ("next 2000") consortium is carrying out a distributed observing campaign with the Keck, Magellan, and Subaru telescopes, as well as the automatic photometric telescopes of Fairborn Observatory, in order to search for short-period gas giant planets around metal-rich stars. We have established a reservoir of more than 14,000 main-sequence and subgiant stars closer than $110 \mathrm{pc}$, brighter than $V=10.5$, and with $0.4<B-V<1.2$. Because the fraction of stars with planets is a sensitive function of stellar metallicity, a broadband photometric calibration has been developed to identify a subset of 2000 stars with $[\mathrm{Fe} / \mathrm{H}]>0.1$ dex for this survey. We outline the strategy and report the detection of a planet orbiting the metal-rich G5 IV star HD 88133 with a period of 3.41 days, semivelocity amplitude $K=35.7 \mathrm{~m} \mathrm{~s}^{-1}$, and $M \sin i=0.29 M_{\mathrm{J}}$. Photometric observations reveal that HD 88133 is constant on the 3.415 day radial velocity period to a limit of 0.0005 mag. Despite a transit probability of $19.5 \%$, our photometry rules out the shallow transits predicted by the large stellar radius.
\end{abstract}

Subject headings: planetary systems: formation — stars: individual (HD 88133)

Online material: machine-readable table

\section{INTRODUCTION}

The Doppler radial velocity (RV) technique is an effective tool that has resulted over the last decade in the detection of 136 extrasolar planets. Ongoing Doppler projects in the U.S. and Europe are currently surveying almost 3000 of the closest and brightest stars $(V<8)$. These surveys now detect Jupiter-like extrasolar planets in wider orbits analogous to our own solar system (Marcy et al. 2002) and Neptune-mass planets with orbital periods of a few days (Butler et al. 2005; McArthur et al. 2004; Santos et al. 2004a, 2004b). Because planets with short orbital periods are so easily detected with Doppler RV measurements, virtually all planets with $M \sin i>0.5 M_{\mathrm{J}}$ and orbital periods less than 14 days (the so-called hot Jupiters) have already been harvested from current Doppler surveys.

\footnotetext{
${ }^{1}$ Based on observations obtained at the W. M. Keck Observatory, which is operated by the University of California and the California Institute of Technology. Keck time has been granted by NOAO and NASA.

2 Department of Physics and Astronomy, San Francisco State University, San Francisco, CA 94132; fischer@stars.sfsu.edu.

${ }^{3}$ UCO/Lick Observatory, University of California at Santa Cruz, Santa Cruz, CA 95064.

4 Department of Terrestrial Magnetism, Carnegie Institute of Washington DC, 5241 Broad Branch Road NW, Washington, DC 20015-1305.

5 Department of Astronomy, University of California, Berkeley, CA 94720.

6 Center of Excellence in Information Systems, Tennessee State University, 330 10th Avenue North, Nashville, TN 37203; and Senior Research Associate, Department of Physics and Astronomy, Vanderbilt University, Nashville, TN 37235.

7 Space Telescope Science Institute, 3700 San Martin Drive, Baltimore, MD 21218.

8 Tokyo Institute of Technology, Ookayama, Meguro-ku, Tokyo 152-8551, Japan; and University of California Observatories, Lick Observatory, University of California, Santa Cruz, CA 95064.

9 Graduate School of Science and Technology, Kobe University, 1-1 Rokkodai, Nada, Kobe 657-8501, Japan.

${ }^{10}$ Department of Astronomy, Pontificia Universidad Catolica, Avenida Vicuña Mackenna 4860, Casilla 306 Santiago 200, Chile.
}

The observation of a hot Jupiter planet transiting the star HD 209458 (Charbonneau et al. 2000, Henry et al. 2000) provided an important direct detection of an extrasolar planet. The quality of physical information for HD 209458b exceeds that of any other known exoplanet because the intrinsic brightness of the host star enables the efficient collection of high-precision $\left(3 \mathrm{~m} \mathrm{~s}^{-1}\right) \mathrm{RV}$ s that allow precise determination of the mass and orbital parameters (e.g., Butler et al. 1996), as well as high temporal resolution Hubble Space Telescope (HST) photometry (Brown et al. 2001) and spectroscopic observations of the atmospheric constituents in the planet atmosphere (Charbonneau et al. 2002; Vidal-Madjar \& Lecavelier des Etangs 2003; VidalMadjar et al. 2004). The intrinsic brightness of HD 209458 also contributed to the precision with which stellar characteristics ( $T_{\text {eff }}$, metallicity, $v \sin i, M_{*}, R_{*}$ ) could be derived; an accurate parallax measurement is available from Hipparcos. Since only the ratio of the planet radius to the stellar radius can be determined, knowledge of the stellar parameters is key to deriving the radius and density of the transiting planet.

The recent spate of discoveries of planets transiting faint stars (Bouchy et al. 2004; Pont et al. 2004; Konacki et al. 2003) indicates that HD 209458b is, in fact, a rather anomalous planet, with a radius $40 \%$ larger than expected from both theory and the radii of other transiting planets. Recently, this result has been greatly strengthened with the exciting detection of a transiting planet around a $V=11.8$ star with a well-determined stellar radius (Alonso et al. 2004; Laughlin et al. 2005). The radius discrepancy observed for HD 209458b has spawned controversy regarding the nature of the mechanism responsible for the planet's bloated condition (Guillot \& Showman 2002; Baraffe et al. 2003; Burrows et al. 2003; Bodenheimer et al. 2003).

Since the detection of HD 209458b, more than 20 photometric transit searches have been started (Horne 2003). Unfortunately, these surveys cannot detect the $90 \%$ of hot Jupiter planets that do not transit their host stars. Nontransiting hot 
Jupiters are important objects in their own right, and the detection of a large number of these objects could provide powerful constraints on theories of planet formation and evolution. For example, these planets can exhibit non-Keplerian interactions with sibling planets on timescales of just a few years. In addition, the eccentricity distribution of short-period planets could reveal whether hot Jupiters have solid cores. The detection of a large number of new hot Jupiters could also shed light on the puzzling concentration of planets in the period range between roughly 2.5 and 3.5 days (nine out of 20 objects with $P<10$ days). This orbital pileup at 3 days is seemingly at odds with the detection of three out of four transiting planets with orbital periods of about 1.5 days (very hot Jupiters) from the OGLE survey (Udalski et al. 2002a, 2002b; Konacki et al. 2003; Bouchy et al. 2004). There is no observational bias against the Doppler detection of $P=1.5$ day planets by the Doppler technique, suggesting that these very hot Jupiters must be much less common than gas giant planets in 3 day orbits.

\section{N2K CONSORTIUM}

Current Doppler surveys have identified about 20 extrasolar planets with orbital periods shorter than 14 days; however, after an initial burst of discoveries, the rate of hot Jupiter detections has trailed off. Virtually all of these easily detected planets have been harvested from current RV surveys. In order to find a substantial number of new hot Jupiters, we have established a consortium of American, Chilean, and Japanese astronomers to carry out a distributed observing program. Using the Keck, Magellan, and Subaru telescopes, we will observe the next 2000 (N2K) closest, brightest, and most metal-rich FGK stars not found in current Doppler surveys.

We first established a reservoir of more than 14,000 mainsequence and subgiant stars drawn from the Hipparcos catalog (ESA 1997). Considerable information is available for this large aggregate of candidate stars, including $B$ and $V$ colors, 2MASS $J H K$ photometry, parallaxes, luminosity, proper motions, photometric variability, and information regarding the presence of companions. Our reservoir stars all have $0.4<B-V<1.2$ and $V<10.5$, and all are closer than $110 \mathrm{pc}$.

The N2K program actively tracks stars as they are drawn out of the reservoir and distributed to a particular telescope for an observing run. At each telescope, we use an iodine cell to provide a wavelength reference spectrum and our standard Doppler pipeline to analyze all stars identically. A spectral synthesis modeling pipeline has also been established at each telescope so that spectroscopic analysis (Valenti \& Fischer 2005) is carried out identically for all stars. Our strategy is to observe a set of star three times over a period of a few days to detect shortperiod RV variations consistent with a hot Jupiter. Monte Carlo simulations show that with a Doppler precision of $7 \mathrm{~m} \mathrm{~s}^{-1}$, more than $90 \%$ of the planets with $M \sin i>0.5 M_{\mathrm{J}}$ and orbital periods between 1.2 and 14 days will show greater than $3 \sigma \mathrm{RV}$ scatter. Spectroscopic binaries typically show rms scatter of several hundred $\mathrm{m} \mathrm{s}^{-1}$ on this timescale and can be immediately dropped. In fact, our Doppler velocities generally have a betterthan-target precision of 4-5 $\mathrm{m} \mathrm{s}^{-1}$, and any stars with significant RV variations obtain immediate RV and photometric follow-up at the telescopes available to the consortium members. Over the next 2 years, the N2K consortium should detect $\sim 60$ new planets with $M \sin i>0.5 M_{\mathrm{J}}$ and orbital periods shorter than 14 days and flag stars having longer period exoplanets.

All stars with significant short-term RV variations will also be observed with the automatic photometric telescopes (APTs) at Fairborn Observatory (Henry 1999; Eaton et al. 2003). As outlined in Butler et al. (2005), the precise photometric measurements from the APTs are useful for establishing whether the $\mathrm{RV}$ variations are caused by photospheric features such as spots and plages or by planetary-reflex motion. In addition, the efficiency and flexibility of the APTs make them ideal for searching for possible transits that would allow the determination of planetary radii and true masses. The high precision of the APT photometry renders transits of hot Jupiters easily visible, even for the subgiant stars in our sample with radii of up to $2.0 R_{\odot}$ (e.g., Henry 2000; see $\S 5$ below).

\subsection{Synthetic Templates}

Over the past 6 months we have been testing the use of synthetic templates for our Doppler analysis pipeline. The advantage of a synthetic template is that it eliminates one RV observation, traditionally taken without iodine. To create the synthetic template we first divided the stellar observation (with I2) by a featureless B star observation (also with I2). This division is never perfect; residual $1 \%$ iodine lines are left in the spectrum, and our first tests showed that this resulted in unacceptable drifts in the wavelength scale as the barycentric velocities change with time. To try to eliminate this velocity drift, we morphed the National Solar Observatory (NSO) solar spectrum (Wallace et al. 1993) to match our iodine-divided stellar spectra. The morphing process involves rotationally broadening the solar absorption lines and then globally rescaling with a pseudo optical depth. Additional fine-tuning is accomplished by multiplying the morphed spectrum by the remaining smoothed residuals. The morphedNSO templates provide reliable short-term RV precision of about $7 \mathrm{~m} \mathrm{~s}^{-1}$ and help to identify spectroscopic binaries that should be dropped from the N2K program. However, there is a slow degradation in precision as the spectral type departs from solar (in $T_{\text {eff }}$, metallicity, or surface gravity). The standard Doppler technique employing an extra template observation still yields the highest precision and is used for stars that warrant long-term RV follow-up.

\section{TARGETING METAL-RICH STARS}

Butler et al. (2001) have shown that $0.75 \%$ of the stars on current Doppler surveys have hot Jupiter companions with orbital periods between 3 and 5 days. However, we expect a higher detection rate because we are exploiting an observed correlation between stellar metallicity and the rate of occurrence of gas giant planets. This correlation has been noted and characterized by Gonzalez (1997, 1998, 1999), Gonzalez et al. (2001), Fuhrmann et al. (1997, 1998), Santos et al. (2000, 2003, 2004a, 2004b), and Reid (2002). Fischer \& Valenti (2005) show that stars with $[\mathrm{Fe} / \mathrm{H}]>0.2$ have at least 3 times as many extrasolar planets as solar metallicity stars, and we will bias our sample with metal-rich stars. In addition, our observing strategy will flag orbital periods out to 14 days, so our detectable parameter space extends beyond the orbits considered to be hot Jupiters by Butler et al. (2001). We expect to find close-in gas giant planets around at least $3 \%$ of the stars surveyed by the $\mathrm{N} 2 \mathrm{~K}$ consortium.

Because the $\mathrm{N} 2 \mathrm{~K}$ program targets metal-rich stars, it is important to note that a statistical analysis that compares stars with planets to a volume-limited sample will not be valid. By construction, planets from this program will be drawn from a much more metal-rich parent population than a volume-limited sample. However, a statistically valid approach is presented in Fischer \& Valenti (2005), in which all observed stars are binned by metallicity, and then the fraction of stars with planets in each metallicity bin is calculated. Adding more metal-rich stars 


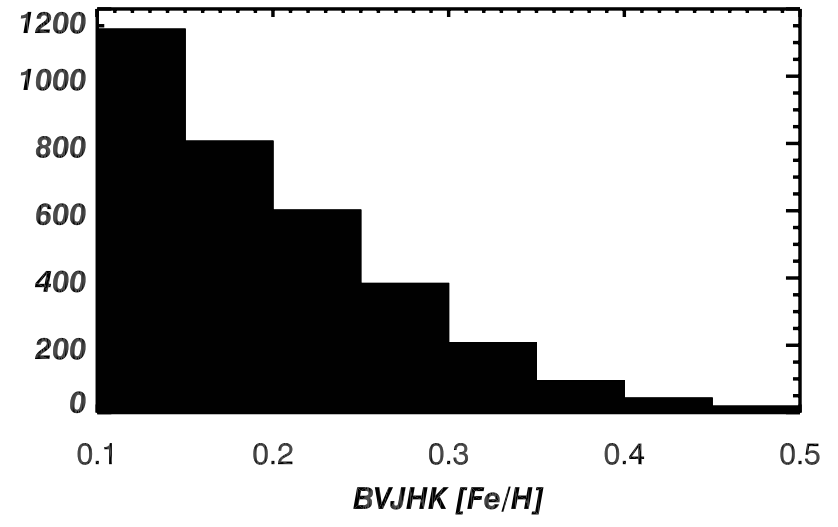

Fig. 1.- Metal-rich tail of the $[\mathrm{Fe} / \mathrm{H}]$ distribution for our N2K reservoir sample of more than 14,000 stars. Broadband photometry $\left(B_{T}, V_{T}, J H K\right)$ was used to estimate the metallicity for all main-sequence and subgiant stars closer than $110 \mathrm{pc}$, brighter than $V=10.5$, with color $0.4<B-V<1.2$, and not on current Doppler programs. The 2000 stars shown here have $[\mathrm{Fe} / \mathrm{H}]>0.1 \mathrm{dex}$ and are the primary targets for the $\mathrm{N} 2 \mathrm{~K}$ program.

to these bins simply decreases the Poisson error bars on the percentage of high-metallicity stars with planets. The lowmetallicity bins will not be statistically incorrect, but they will have large uncertainties until surveys of metal-poor stars provide better statistics.

Of key importance to the success of this project, a photometric calibration using broadband filters was developed (Ammons et al. 2005) to provide $T_{\text {eff }}$ and metallicity estimates for every star in our reservoir using Tycho $B V$ (Hog et al. 2000), 2MASS $J H K$ photometry (Cutri et al. 2003), and proper motions. Our ability to produce a Hipparcos-2MASS metallicity calibration was made possible by our possession of a high-quality "training set" of spectroscopic metallicities for more than 1100 FGK stars (Valenti \& Fischer 2005). Figure 1 shows a histogram of the subset of supersolar metallicity stars from our reservoir sample. This set of more than 2000 stars with $[\mathrm{Fe} / \mathrm{H}]>0.1 \mathrm{dex}$ comprises the core target sample for the $\mathrm{N} 2 \mathrm{~K}$ program. We are also obtaining low-resolution spectroscopy with the Gold Spectrograph on the $2.1 \mathrm{~m}$ Kitt Peak telescope and the Nickel spectrograph at Lick Observatory to verify the broadband photometry metallicity calibration. Low-resolution equivalent width measurements for a set of spectral lines have been calibrated

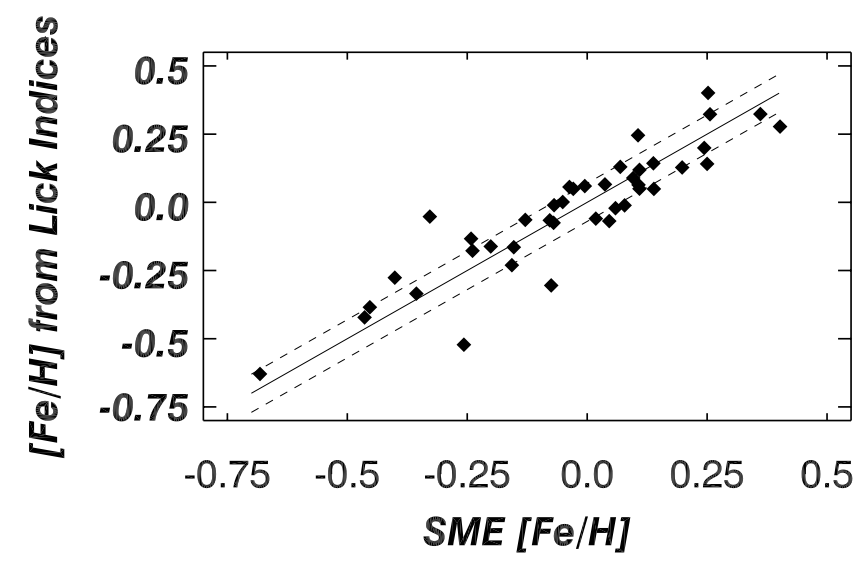

FIG. 2.-Lick indices based on equivalent widths of a set of low-resolution spectral lines have been calibrated to metallicities derived from spectral synthesis modeling of high-resolution spectra using SME; they show agreement to better than 0.1 dex.

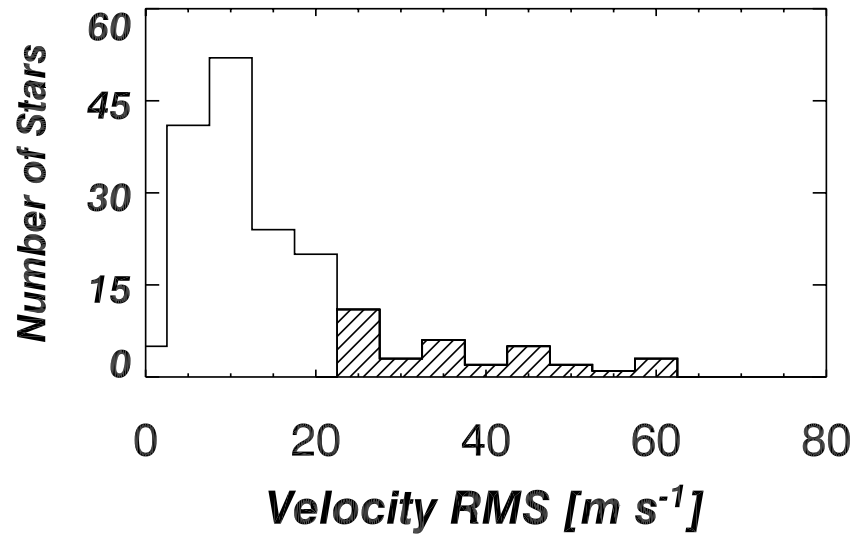

FIG. 3.-Velocity scatter less than $80 \mathrm{~m} \mathrm{~s}^{-1}$ is observed for 175 of 211 stars observed at Keck after three or more RV observations. RV scatter less than $3 \sigma$ is seen for 148 of these stars. However, 35 stars, or $16 \%$ of the observed sample (indicated in the hatched bins), show greater than $3 \sigma \mathrm{RV}$ scatter, consistent with the presence of a hot Jupiter. The planet announced in this paper had an initial velocity rms of $24 \mathrm{~m} \mathrm{~s}^{-1}$.

(S. E. Robinson et al. 2005, in preparation) using the same training set of spectroscopic metallicities (Valenti \& Fischer 2005). These indices provide a spectroscopic abundance that agrees to better than 0.1 dex (Fig. 2).

\section{THE FIRST N2K RUN}

The first set of N2K stars was observed during a three night observing run at Keck in the 2004A semester. After a single observation on the first night, we determined the chromospheric activity of the observed stars (Wright et al. 2004, Baliunas et al. 1997, Noyes et al. 1984), and we ran the spectra through our spectral synthesis pipeline to determine metallicity, $\log g$, and $v \sin i$, and to identify double-lined spectroscopic binaries (SB2s). As a result of this "morning after" screening, 12 stars were identified as SB2s or rapid rotators and were dropped before a second observation was obtained. All information regarding every observed star, including RV measurements, information from spectral synthesis modeling, information about the presence of stellar companions, and chromospheric activity measurements will appear in a subsequent catalog paper.

We obtained three or more RV measurements for 211 stars at Keck in the 2004A semester with a typical precision of $4 \mathrm{~m} \mathrm{~s}^{-1}$ for stars with standard templates and $7 \mathrm{~m} \mathrm{~s}^{-1}$ for stars with

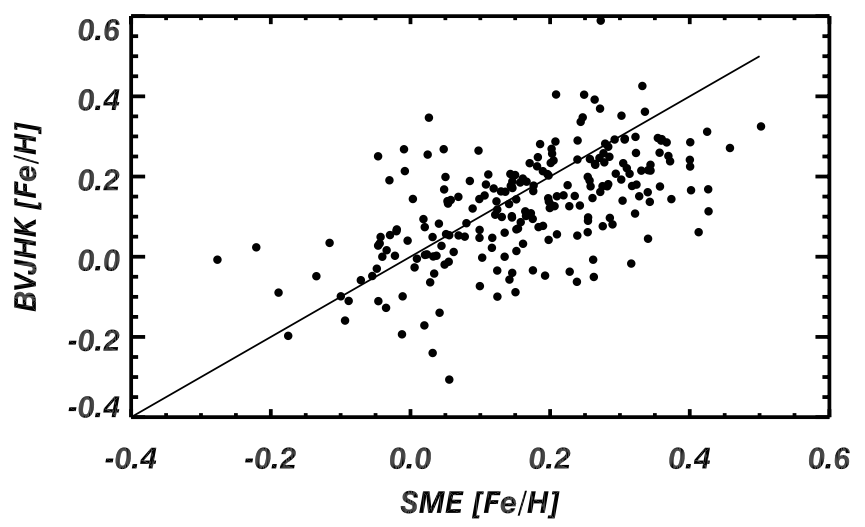

FIG. 4.-Comparison of spectroscopically derived $[\mathrm{Fe} / \mathrm{H}]$ with the metallicity estimate from our BVJHK calibration (Ammons et al. 2005) for 211 stars observed at Keck. The solid line shows a 1:1 correlation. 
TABLE 1

Stellar Parameters for HD 88133

\begin{tabular}{|c|c|}
\hline Parameter & Value \\
\hline$V \ldots$ & 8.01 \\
\hline$M_{V} \ldots \ldots$ & 3.65 \\
\hline$B-V \ldots \ldots$ & 0.81 \\
\hline Spectral type & G5 IV \\
\hline Distance $(\mathrm{pc})$ & 74.46 \\
\hline$[\mathrm{Fe} / \mathrm{H}] \ldots \ldots \ldots$ & $0.34 \pm 0.04$ \\
\hline$T_{\text {eff }}(\mathrm{K})$ & $5494 \pm 23$ \\
\hline$v \sin i \mathrm{~km} \mathrm{~s}^{-1}$ & $2.2 \pm 0.3$ \\
\hline $\log g$ & $4.23 \pm 0.05$ \\
\hline$M_{\text {star }}\left(M_{\odot}\right)$ & $1.2 \pm 0.2$ \\
\hline$R_{\text {star }}\left(R_{\odot}\right)$ & $1.93 \pm 0.06$ \\
\hline$S_{H K}$ & 0.138 \\
\hline $\log R_{H K}^{\prime}$ & -5.16 \\
\hline 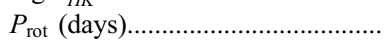 & 48.0 \\
\hline
\end{tabular}

synthetic templates. The RV variations fell into three categories: (1) 148 stars showed less than $3 \sigma \mathrm{RV}$ scatter and were retired from the N2K program, (2) 19 stars were spectroscopic binaries with RV variations greater than $500 \mathrm{~m} \mathrm{~s}^{-1}$ (these spectroscopic binaries were also retired from the N2K program), and (3) 35 stars (16\% of the 211 star sample) showed rms scatter greater than $3 \sigma$ consistent with the presence of a shortperiod planet.

The distribution of rms scatter for these stars is shown in Figure 3. The hatched bins in Figure 3 represent the 35 stars having RV scatter between 20 and $80 \mathrm{~m} \mathrm{~s}^{-1}$. These are the stars that warrant RV follow-up to search for short-period planets. One of these stars, HD 88133, showed an initial rms scatter of $24 \mathrm{~m} \mathrm{~s}^{-1}$. This star now has enough RV measurements to characterize an orbiting hot Jupiter planet, as described below.

Spectral synthesis modeling was carried out for all 211 stars yielding $[\mathrm{Fe} / \mathrm{H}], T_{\text {eff }}, \log g$, and $v \sin i$ with uncertainties of $0.04 \mathrm{dex}, 23 \mathrm{~K}, 0.05 \mathrm{dex}$, and $0.3 \mathrm{~m} \mathrm{~s}^{-1}$, respectively, as discussed in Valenti \& Fischer (2005). Figure 4 shows that the spectroscopic metallicities agree well with the broadband metallicity estimates (Ammons et al. 2005), confirming that

TABLE 2

Radial Velocities For HD 88133

\begin{tabular}{|c|c|c|}
\hline JD -2440000 & $\begin{array}{c}\mathrm{RV} \\
\left(\mathrm{m} \mathrm{s}^{-1}\right)\end{array}$ & $\begin{array}{c}\text { Uncertainties } \\
\quad\left(\mathrm{m} \mathrm{s}^{-1}\right)\end{array}$ \\
\hline $13014.948 \ldots \ldots$. & -14.3 & 4.09 \\
\hline $13015.947 \ldots \ldots \ldots \ldots \ldots \ldots \ldots \ldots$ & 36.9 & 3.99 \\
\hline 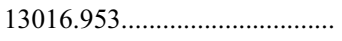 & 29.8 & 4.22 \\
\hline 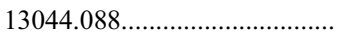 & 35.8 & 3.92 \\
\hline $13044.869 \ldots \ldots \ldots \ldots \ldots \ldots \ldots \ldots . .$. & -14.6 & 3.18 \\
\hline 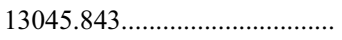 & -20.7 & 2.98 \\
\hline $13046.081 \ldots \ldots \ldots \ldots \ldots \ldots \ldots$ & -11.9 & 2.94 \\
\hline 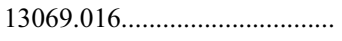 & -23.1 & 4.35 \\
\hline $13071.788 \ldots \ldots \ldots \ldots \ldots \ldots \ldots$ & 13.8 & 3.82 \\
\hline $13072.021 \ldots \ldots \ldots \ldots \ldots \ldots \ldots$ & -9.2 & 4.26 \\
\hline $13073.950 \ldots \ldots \ldots \ldots \ldots \ldots \ldots \ldots$ & 23.7 & 4.66 \\
\hline $13076.948 \ldots \ldots \ldots \ldots \ldots \ldots \ldots \ldots$ & 0.0 & 6.38 \\
\hline $13153.760 \ldots \ldots \ldots \ldots \ldots \ldots \ldots$ & 17.3 & 3.25 \\
\hline 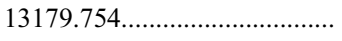 & 20.2 & 3.59 \\
\hline 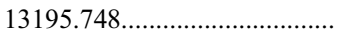 & -19.2 & 2.86 \\
\hline $13197.762 \ldots$ & 33.5 & 3.27 \\
\hline $13199.751 \ldots \ldots \ldots \ldots \ldots \ldots \ldots \ldots \ldots . .$. & -5.6 & 3.36 \\
\hline
\end{tabular}

TABLE 3

Orbital Parameters for HD $88133 \mathrm{~b}$

\begin{tabular}{|c|c|}
\hline Parameter & Value \\
\hline$P$ (days) & $3.415 \pm 0.001$ \\
\hline$T_{p}(\mathrm{JD})$ & $2453016.4 \pm 1.2$ \\
\hline$\omega(\operatorname{deg})$ & $10.2 \pm 162.9$ \\
\hline 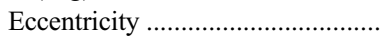 & $0.11 \pm 0.05$ \\
\hline$K_{1}\left(\mathrm{~m} \mathrm{~s}^{-1}\right)$ & $35.7 \pm 2.2$ \\
\hline$a(\mathrm{AU})$ & 0.046 \\
\hline$a_{1} \sin i(\mathrm{AU})$ & $1.11 \mathrm{E}-05$ \\
\hline$f_{1}(m)\left(M_{\odot}\right)$ & $1.58 \mathrm{E}-11$ \\
\hline$M \sin i\left(M_{\mathrm{J}}\right) \ldots \ldots \ldots \ldots \ldots \ldots \ldots \ldots \ldots$ & 0.29 \\
\hline$N_{\text {obs }}$ & 17 \\
\hline $\mathrm{rms}\left(\mathrm{m} \mathrm{s}^{-1}\right)$ & 5.3 \\
\hline Reduced $\sqrt{\chi_{\nu}^{2}} \ldots \ldots \ldots \ldots \ldots \ldots \ldots \ldots \ldots \ldots$ & 1.27 \\
\hline
\end{tabular}

the broadband photometric calibration is an excellent way to identify metal-rich target stars for the N2K program.

\section{HD 88133}

HD 88133 is a G5 IV star with $V=8.0$ and $B-V=0.81$. The Hipparcos parallax (ESA 1997) of 13.43 mas places the star at a distance of $74.5 \mathrm{pc}$ with an absolute visual magnitude, $M_{V}=$ 3.65 . Our spectroscopic analysis yields $T_{\text {eff }}=5494 \pm 23 \mathrm{~K}$, $[\mathrm{Fe} / \mathrm{H}]=0.34 \pm 0.04, \log g=4.23 \pm 0.05$, and $v \sin i=2.2 \mathrm{~km}$ $\mathrm{s}^{-1}$. From the bolometric luminosity and $T_{\text {eff }}$ we derive a stellar radius of $1.93 R_{\odot}$, and evolutionary tracks of Girardi et al. (2000) provide a stellar mass estimate using SME (spectroscopy made easy; Valenti \& Piskunov 1996) of $1.2 M_{\odot}$. The chromospheric activity of HD 88133 is $S_{H K}=0.138$ and $\log R_{H K}^{\prime}=-5.16$. Using the relation between rotation period and the $S_{H K}$ index (Noyes et al. 1984), we estimate a rotational period of 48 days for this star. Stellar parameters are summarized in Table 1.

The observation dates, RVs, and RV uncertainties for HD 88133 are listed in Table 2. We estimate the stellar jitter for this star to be $3.2 \mathrm{~m} \mathrm{~s}^{-1}$ and add this jitter in quadrature with the formal RV errors when fitting the data with a Keplerian and calculating $\left(\chi_{\nu}^{2}\right)^{1 / 2}$. Our best-fit orbital parameters are listed in Table 3, and the Keplerian fit is overplotted on the phased RV data in Figure 5. The orbital period is 3.41 days, with a velocity semiamplitude of $35.7 \mathrm{~m} \mathrm{~s}^{-1}$ and eccentricity of $0.11 \pm 0.05$ with rms scatter of $5.26 \mathrm{~m} \mathrm{~s}^{-1}$ and $\left(\chi_{\nu}^{2}\right)^{1 / 2}$ of 1.26 . With the

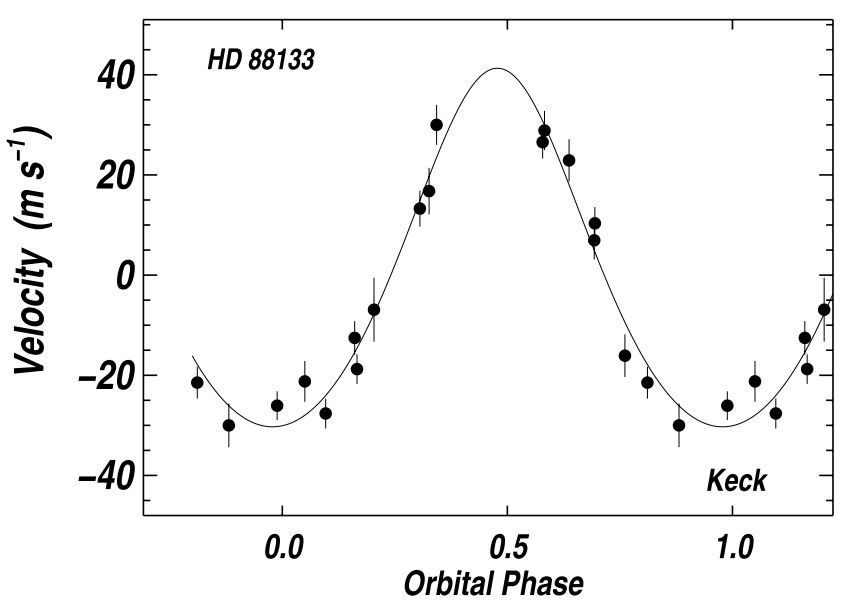

FIG. 5.-Phased RVs for HD 88133. With an orbital period of 3.41 days, velocity amplitude of $35.7 \mathrm{~m} \mathrm{~s}^{-1}$, and stellar mass of $1.2 M_{\odot}$, we derive a planet mass $M \sin i=0.29 M_{\mathrm{J}}$ and orbital radius of $0.046 \mathrm{AU}$. 
TABLE 4

Photometric Observations of HD 88133

\begin{tabular}{|c|c|}
\hline JD $-2,400,000$ & $(b+y) / 2$ \\
\hline $53064.6466 \ldots$ & 1.5043 \\
\hline $53064.6537 \ldots$ & 1.5012 \\
\hline $53064.6701 \ldots$ & 1.5027 \\
\hline $53066.6451 \ldots$. & 1.5074 \\
\hline $53066.6623 \ldots$ & 1.5031 \\
\hline 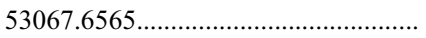 & 1.5024 \\
\hline
\end{tabular}

Note.-Table 4 is published in its entirety in the electronic edition of the Astrophysical Journal. A portion is shown here for guidance regarding its form and content.

stellar mass of $1.2 M$, we derive $M \sin i=0.29 M_{\mathrm{J}}$ and $a_{\mathrm{rel}}=$ 0.046 AU. Uncertainties were estimated by running 100 Monte Carlo trials, fitting in the orbital parameters listed in Table 3. The models of Bodenheimer et al. (2003) predict a planetary radius for HD $88133 \mathrm{~b}$ of $0.97 R_{\mathrm{J}}$ if the planet has a core and $1.12 R_{\mathrm{J}}$ if it does not.

The best-fit eccentricity of 0.11 is unusual, since giant planets with orbital periods shorter than 5 days are expected to be tidally circularized. Fixing the eccentricity to zero yields a slightly increased $\left(\chi_{\nu}^{2}\right)^{1 / 2}$ of 1.48 and an rms to the fit of $6.3 \mathrm{~m} \mathrm{~s}^{-1}$. Again, we have added jitter in quadrature to the formal RV errors. This constitutes an acceptable fit; however, this star warrants additional RV follow-up to determine the eccentricity more accurately and to check for any additional planetary companions.

In addition to the RV observations, we have collected 161 photometric measurements (see Table 4) between 2004 March and June with the T12 $0.8 \mathrm{~m}$ APT at Fairborn Observatory. The transit probability is given by the ratio of the stellar radius to the semimajor axis of the orbit: $R_{*} / a=19.5 \%$. The telescope, photometer, observing procedures, and data reduction techniques are described briefly in Butler et al. (2005 and references therein). Our photometric comparison star was HD 88270 ( $V=6.64, B-V=0.36, \mathrm{~F} 2 \mathrm{~V})$, which has been shown to be constant to 0.002 mag or better from intercomparison with additional comparison stars.

The 161 combined $(b+y) / 2$ differential magnitudes of HD 88133 were phased with the planetary orbital period and a time of inferior conjunction (computed from the orbital elements in Table 3) and plotted in Figure 6. The standard deviation of the observations is $0.0029 \mathrm{mag}$, slightly larger than the typical precision with this telescope, most likely because HD 88133 is somewhat fainter than most program stars. Period analysis does not reveal evidence of any periodicity between 1 and 60 days. A least-squares sine fit of the observations phased to the RV period gives a semiamplitude of $0.00054 \pm$ $0.00030 \mathrm{mag}$, so starspots are unlikely to be the cause of the RV variations. The solid curve in Figure 6 approximates the predicted transit light curve (assuming central transits) computed from the orbital elements, the stellar radius, and an adopted planetary radius equal to Jupiter's. The horizontal bar below the predicted transit window represents the approximate uncertainty in the time of mid-transit, based on Monte Carlo simulations and the uncertainties in the orbital elements. The predicted transit depth is only $0.0031 \mathrm{mag}$ because of the large stellar radius. However, the mean of the eight observations within the transit window agrees with the mean of the $153 \mathrm{ob}-$ servations outside the window to within 0.0005 mag, just as expected from the precision of the observations. Thus, central (nongrazing) transits are ruled out by our photometry.

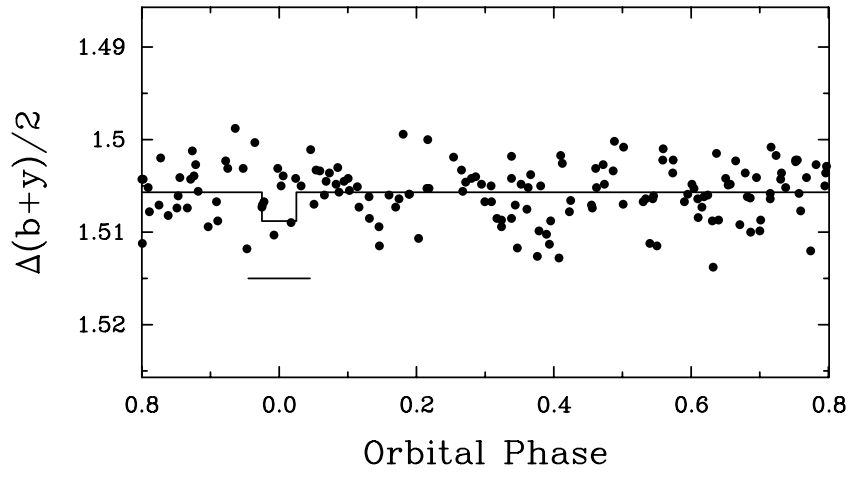

FIG. 6.-Strömgren $(b+y) / 2$ photometric observations of HD 88133 acquired with the T12 $0.8 \mathrm{~m}$ APT at Fairborn Observatory and phased to the RV period. There is no evidence in the observations for any periodicity between 1 and 100 days. The star is constant on the RV period to a limit of $0.0005 \mathrm{mag}$, supporting the planetary interpretation of the RV variations. Although predicted transit depths are only 0.003 mag because of the large stellar radius, such transits are nonetheless ruled out by the photometry.

\section{DISCUSSION}

The N2K consortium was established to survey "the next 2000" closest and brightest high-metallicity stars using a distributed observing program and a quick-look strategy to identify stars with rms velocity scatter consistent with the presence of a hot Jupiter companion. Over the next 2 years, this program should identify 60 new gas giant planets in short-period orbits, quadrupling the number of hot Jupiters that have been discovered and providing six new transiting planets around stars brighter than $V=10.5$.

Observations of the first 211 stars at Keck have identified 35 stars with velocity variations consistent with the presence of an extrasolar planet. Follow-up RV observations on one of these stars (HD 88133) has confirmed the presence of a planet in a 3.41 day orbit with $M \sin i=0.29 M_{\mathrm{J}}$. Our photometric observations have established that HD 88133 is highly constant on the RV period and that it does not undergo transits at the predicted times.

Spectroscopic analysis of the program stars requires about $1 \mathrm{CPU}$ hr to run, so it is possible to analyze $\sim 300$ stars in one $24 \mathrm{hr}$ period on our computer cluster at San Francisco State University. This pipeline analysis makes it possible to check every star the morning after the first observation is obtained. Unsuitable stars (SB2s, rapid rotators) are immediately dropped, and the observing list is backfilled with new stars.

In addition to the 250 stars observed in the 2004A semester at Keck, we have completed three or more observations of 130 stars at Subaru. All of these stars have spectroscopic and Doppler analysis completed, and we are obtaining follow-up observations on those stars with rms velocity scatter greater than $3 \sigma$ and less than $100 \mathrm{~m} \mathrm{~s}^{-1}$. At Magellan, 90 stars have multiple observations, and we are beginning the Doppler analysis of those stars. Thus, the first 500 of 2000 metal-rich stars are now beginning to be processed by the $\mathrm{N} 2 \mathrm{~K}$ consortium.

We gratefully acknowledge the dedication and support of the Keck Observatory staff. We thank the NOAO and NASA telescope assignment committees for generous allocations of telescope time. We thank the Michaelson Science Center for travel support and support through the KDPA program. G. W. H. acknowledges support from NASA grant NCC5-511 and NSF 
grant HRD-9706268. We acknowledge support by NASA grant NAG5-75005 (to G. W. M.), NSF grant AST-9988358, NASA grant NAG5-4445 (to S. S. V.), and NASA grant NNG04GKi9G (to G. L.). D. M. is supported by FONDAP 15010003 . We are grateful to Sun Microsystems for ongoing support. We thank the anonymous referee for helpful comments. This research has made use of the Simbad database, operated at CDS, Strasbourg, France. The authors extend thanks to those of native Hawaiian ancestry on whose sacred mountain of Mauna Kea we are privileged to be guests. Without their generous hospitality, the Keck observations presented herein would not have been possible.
Alonso, R., et al. 2004, ApJ, 613, L153

Ammons, M., et al. 2005, ApJ, submitted

Baliunas, S. L., Henry, G. W., Donahue, R. A., Fekel, F. C., \& Soon, W. H. 1997, ApJ, 474, L119

Baraffe, I., Chabrier, G., Barman, T. S., Allard, F., \& Hauschild, P. H. 2003, A\&A, 402, 701

Bodenheimer, P., Laughlin, G., \& Lin, D. N. C. 2003, ApJ, 592, 555

Bouchy, F., Pont, F., Santos, N. C., Melo, C., Mayor, M., Queloz, D., \& Udry, S. 2004, A\&A, 421, L13

Brown, T. M., Charbonneau, D., Gilliland, R. L., Noyes, R. W., \& Burrows, A. 2001, ApJ, 552, 699

Burrows, A., Sudarsky, D., \& Hubbard, W. B. 2003, ApJ, 594, 545

Butler, R. P., Marcy, G. W., Williams, E., McCarthy, C., Dosanjh, P., \& Vogt, S. S. 1996, PASP, 108, 500

Butler, R. P., Tinney, C. G., Marcy, G. W., Jones, H. R. A., Penny, A. J., \& Apps, K. 2001, ApJ, 555, 410

Butler, R. P., Vogt, S. S., Marcy, G. W., Fischer, D. A., Wright, J. T., Henry, G. W., Laughlin, G., \& Lissauer, J. 2005, ApJ, submitted

Charbonneau, D., Brown, T. M., Latham, D. W., \& Mayor, M. 2000, ApJ, 529, L45

Charbonneau, D., Brown, T. M., Noyes, R. W., \& Gilliland, R. L. 2002, ApJ, 568,377

Cutri, R. M., et al. 2003. VizieR Online Data Catalog, II/246 (Cambridge: Cambridge Univ. Press)

Eaton, J. A., Henry, G. W., \& Fekel, F. C. 2003, in The Future of Small Telescopes in the New Millennium, Volume II-The Telescopes We Use, ed. T. D. Oswalt (Dordrecht: Kluwer), 189

ESA. 1997, The Hipparcos and Tycho Catalogues (ESA-SP 1200; Noordwijk: ESA)

Fischer, D. A., \& Valenti, J. A. 2005, ApJ, in press

Fuhrmann, K., Pfeiffer, M. J., \& Bernkopf, J. 1997, A\&A, 326, 1081 1998, A\&A, 336, 942

Girardi, L, Bressan, A. Bertelli, G., \& Chiosi, C. 2000, A\&AS, 141, 371

Gonzalez, G. 1997, MNRAS, 285, 403

1998, A\&A, 334, 221

1999, MNRAS, 308, 447

\section{REFERENCES}

Gonzalez, G., Laws, C., Tyagi, S., \& Reddy, B. E. 2001, AJ, 121, 432

Guillot, T., \& Showman, A. P. 2002, A\&A, 385, 156

Henry, G. W. 1999, PASP, 111, 845 2000, ApJ, 536, L47

Henry, G. W., Marcy, G. W., Butler, R. P., \& Vogt, S. S. 2000, ApJ, 529, L41 Hog, E., et al. 2000, A\&A, 355, L27

Horne, K. 2003, in ASP Conf. Ser. 294, Scientific Frontiers of Planetary Transit Searches: Hot Jupiters Galore, ed. D. Deeming \& S. Seager (San Francisco: ASP), 361

Konacki, M., Torres, G., Sasselov, D. D., \& Jha, S. 2003, ApJ, 597, 1076

Laughlin, G., Wolf, A., Vanmunster, T., Bodenheimer, P., Fischer, D., Marcy, G., Butler, P., \& Vogt, S. 2005, ApJ, submitted

Marcy, G. W., Butler, R. P., Fischer, D. A., Laughlin, G., Vogt, S. S., Henry, G. W., \& Pourbaix, D. 2002, ApJ, 581, 1375

McArthur, B. E., et al. 2004, ApJ, 614, L81

Noyes, R. W., Hartmann, L., Baliunas, S. L., Duncan, D. K., \& Vaughan, A. H. 1984, ApJ, 279, 763

Pont, F., Bouchy, F., Queloz, D., Santos, N. C. Melo, C., Mayor, M., \& Udry, S. 2004, A\&A, 462, L15

Reid, I. N. 2002, PASP, 114, 306

Santos, N. C., Israelian, G., \& Mayor, M. 2000, A\&A, 363, 228 2004a, A\&A, 415, 1153

Santos, N. C., Israelian, G., Mayor, M., Rebolo, R., \& Udry, S. 2003, A\&A, 398,363

Santos, N. C., et al. 2004b, A\&A, 426, L19

Udalski, A., et al. 2002a, Acta Astron., 52, 1 2002b, Acta Astron., 52, 115

Valenti, J. A., \& Fischer, D. A. 2005, ApJ, submitted

Valenti, J., \& Piskunov, N. 1996, ARAS, 118, 595

Vidal-Madjar, A., \& Lecavelier des Etangs, A. 2005, in ASP Conf. Ser. 321, Extrasolar Planets: Today and Tomorrow, ed. J.-P. Beaulieu, A. Lecavelier des Etangs, \& C. Terquem (San Francisco: ASP), in press

Vidal-Madjar, A., Désert, J.-M., Lecavelier des Etangs, A., Hébrard, G., Ballester, G. E., Ehrenreich, D., \& Ferlet, R. 2004, ApJ, 604, L69

Wallace, L., Hinkle, K., \& Livingston, W. C. 1993, NSO Tech. Rep. 93-001

Wright, J. T., Marcy, G. W., Butler, R. P., \& Vogt, S. S. 2004, ApJS, 152, 261 